\title{
Analytical approach to integrate the different components of direct tunneling current through ultrathin gate oxides in $n$-channel metal-oxide-semiconductor field-effect transistors
}

\author{
Kingsuk Maitra and Navakanta Bhat* \\ Department of Electrical Communication Engineering, Indian Institute of Science, Bangalore-560012, India
}

\begin{abstract}
An analytical scheme to combine the channel component and the edge component of direct tunneling current through ultrathin gate oxides in $n$-channel metal-oxide-semiconductor field-effect transistors has been developed. The results obtained have been calibrated against the published experimental and numerical simulation data. The inherent simplicity of the proposed analytical model makes it suitable for implementing in circuit simulators. The proposed model is capable of predicting the tunneling current under positive as well as negative gate bias. The impact of gate-source/drain extension overlap length (edge) on total gate leakage is clearly quantified. It is demonstrated that the overlap length should be scaled to decrease the leakage power consumption and also to extend the scalability of gate oxide to lower values.
\end{abstract}

\section{INTRODUCTION}

The edge direct tunneling (EDT) current form the gate overlap region into the source drain extension (SDE) region has been identified as the principal source of off-state power dissipation in state-of-the-art very large scale integration (VLSI) chips. ${ }^{1}$ By taking a simple two stage cascaded inverter circuit, the importance of the EDT component of tunneling leakage was demonstrated in the total gate power consumption. We reiterate that for sub-100 nm devices, more than half of the total gate power dissipation comes from the EDT component. ${ }^{1}$ Yang et al. ${ }^{2}$ have also shown that the EDT component of gate leakage exceeds even band-to-band tunneling (BTBT) and gate induced drain leakage (GIDL) for ultrathin gate oxides in metal-oxide-semiconductor fieldeffect transistors (MOSFETs).

Numerical techniques to model the EDT component have been developed. ${ }^{1-3}$ Furthermore, the numerical modeling is restricted to EDT in the subflatband regime for negative gate voltage. However, in light of the fact that gate leakage also influences the functionality of circuits, a simple yet accurate framework which predicts the tunneling current trends under positive as well as negative applied gate bias is necessary. In order to ensure reasonable accuracy, it is necessary to consider both EDT and the channel component (tunneling between the substrate and the gate region) of direct tunneling leakage. The schematic in Fig. 1 illustrates the different components of tunneling leakage mentioned above.

The purpose of this work is to integrate the two components in a simple analytical model. Based on an earlier published $\operatorname{model}^{4}$ on channel components, we have been able to integrate both the edge component and channel component of direct tunneling fully analytically for the first time. We have tuned the existing fitting parameter in Ref. 4 , i.e., $\alpha$, for

*Electronic mail: navakant@ece.iisc.ernet.in the channel and the overlap region to obtain a match with the experimental data. The proposed model is able to predict the tunneling leakage current fairly accurately under both positive and negative gate bias. Further we have shown that for the sub-100 nm technology the overlap length cannot be kept constant at $15-20 \mathrm{~nm}$ as suggested in the literature. ${ }^{5}$ In the context of gate leakage, the oxide scaling limit for $1 \mathrm{nA} / \mu \mathrm{m}$ leakage is shown to be a function of overlap length.

The article is organized as follows. In the next section, the analytical modeling technique used in this work is explained in detail. The subsequent section presents the model predictions in comparison with the experimental data. The Conclusion section highlights the contributions made in this article.

\section{MODELING}

Analytical techniques to model the direct tunneling current have largely been based on the WKB approximation. ${ }^{6}$ More involved physical models for direct tunneling current from the poly-Si gate into the substrate for negative $V_{g}$ in nMOSFETs have been developed in Ref. 7. A physics based modified WKB formalism has been developed which has been extended suitably to predict EDT. ${ }^{2,8}$ A consistent model covering the entire bias range has been published very recently ${ }^{4}$ by rectifying the discrepancies that were present in the original WKB formalism ${ }^{6}$ by introducing a few empirical parameters. The robustness of the model in Ref. 4 has been clearly demonstrated by calibrating it with a large number of experimental and numerical data. We adopt this model to find the gate leakage component from the channel and the overlap region separately. Different $\alpha$ values for the channel and the overlap region $[\alpha($ channel $)=0.6$ and $\alpha$ (overlap) $=0.27$ ] have been used to obtain an overall best fit with experimental results reported in Ref. 1. An effective mass $m_{\text {ox }}$ value of $0.55 m_{0}$ has been used throughout the calculation. The oxide thickness, $T_{\text {ox }}$, refers to the physical oxide 


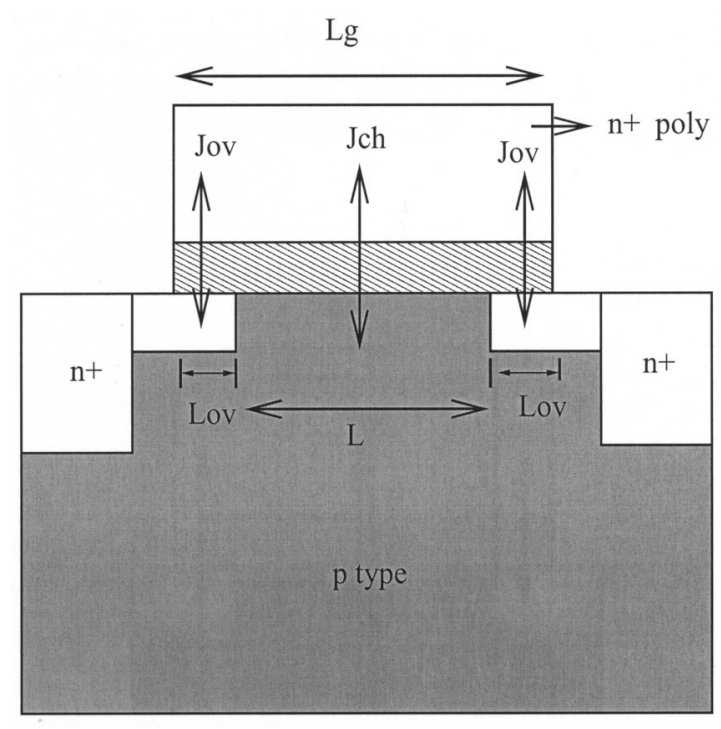

FIG. 1. Schematic showing the edge and the channel component of direct tunneling in nMOSFETs.

thickness. The drain to source voltage $V_{\mathrm{ds}}$ is assumed to be zero. Below we give the steps which are new to our scheme.

The total gate current density is modeled as follows:

$$
J_{\mathrm{tot}}=\frac{J_{\mathrm{ch}} L+2 J_{\mathrm{ov}} L_{\mathrm{ov}}}{L_{g}}
$$

where $L_{g}$ is the total gate length, $L_{\mathrm{ov}}$ the overlap length, and $L$ is the effective gate length such that $L=\left(L_{g}-2 L_{\mathrm{ov}}\right)$.

Each of the components $J_{\mathrm{ch}}$ and $J_{\mathrm{ov}}$ are modeled as follows:

$$
J_{\mathrm{ch}(\mathrm{ov})}=A C_{\mathrm{ch}(\mathrm{ov})} T_{\mathrm{ch}(\mathrm{ov})},
$$

where $A=q^{3} / 8 \pi h \phi_{b}, T$ is the WKB transmission probability given as follows:

$T_{\mathrm{ch}(\mathrm{ov})}=\exp \left(\frac{-8 \pi \sqrt{2 m_{\mathrm{ox}}} \phi_{b}^{3 / 2}\left[1-\left(1-\frac{\left|V_{\text {oxch(ov) }}\right|}{\phi_{b}}\right)^{3 / 2}\right]}{3 h q\left|E_{\text {oxch }(\mathrm{ov})}\right|}\right)$,

and $C_{\mathrm{ch}(\mathrm{ov})}$ is the correction factor introduced in Ref. 4 which is updated keeping in mind the fact that its value will be different for the channel and the overlap region. Hence it is modified as follows:

$$
\begin{aligned}
C_{\mathrm{ch}(\mathrm{ov})}= & \exp \left[\frac{20}{\phi_{b}}\left(\frac{\left|V_{\text {oxch }(\mathrm{ov})}\right|-\phi_{b}}{\phi_{b o}}+1\right)^{\alpha}\right. \\
& \left.\times\left(1-\frac{\left|V_{\text {oxch(ov) })}\right|}{\phi_{b}}\right)\right] \frac{V_{g}}{T_{\mathrm{ox}}} N_{\mathrm{ch}(\mathrm{ov})},
\end{aligned}
$$

where $\phi_{b}=\phi_{b o}=3.1 \mathrm{eV}, V_{\text {ox }}$ is the voltage across the gate oxide. The reason for using the different values of $C$ for the channel and overlap region is explained in the following discussion.

(1) $N$ for channel and overlap region.

$$
\begin{aligned}
& N_{\text {channel }}=\left\{\begin{array}{l}
\frac{\varepsilon_{\text {ox }}}{T_{\text {ox }}}\left\{n_{\text {acc }} v_{t} \cdot \log \left[1+\exp \left(-\frac{\left(V_{g}-V_{\mathrm{FB}}\right)}{n_{\mathrm{acc}} v_{t}}\right)\right]\right\} \\
\text { for } V_{g}<0 \\
\frac{\varepsilon_{\mathrm{ox}}}{T_{\mathrm{ox}}}\left\{n_{\mathrm{inv}} v_{t} \cdot \log \left[1+\exp \left(\frac{\left(V_{g e}-V_{\mathrm{th}}\right)}{n_{\mathrm{inv}} v_{t}}\right)\right]\right\}, \\
\text { for } V_{g}>0
\end{array}\right. \\
& N_{\text {overlap }}=\left\{\begin{array}{l}
\frac{\varepsilon_{\mathrm{ox}}}{T_{\mathrm{ox}}}\left\{n_{\mathrm{acc}} v_{t} \cdot \log \left[1+\exp \left(-\frac{V_{g}}{n_{\mathrm{acc}} v_{t}}\right)\right]\right\} \\
\text { for } V_{g}<0 \\
\frac{\varepsilon_{\mathrm{ox}}}{T_{\mathrm{ox}}}\left\{n_{\mathrm{acc}} v_{t} \cdot \log \left[1+\exp \left(\frac{V_{g e}}{n_{\mathrm{acc}} v_{t}}\right)\right]\right\} \\
\text { for } V_{g}>0
\end{array}\right.
\end{aligned}
$$

It is to be noted that for the overlap region $V_{\mathrm{FB}}$ is almost zero, given the fact that both the SDE region and the overlying poly-Si region are heavily doped $n^{+}$regions. In the following discussion, we use $V_{\mathrm{FB}}$ for channel flatband voltage.

The difference in availability of mobile electrons for tunneling in the subflatband region in the channel and overlap region for $V_{g}<0$ is ensured by the induction of the $V_{\mathrm{FB}}$ term in Eq. (5) above.

Following Ref. 4, the impact of poly depletion for $V_{g}$ $>0$ is lumped into $V_{g e}=\left(V_{g}-V_{\text {poly }}\right)$ replacing substrate doping concentration $N_{\text {sub }}$ with SDE doping concentration $N_{\text {ov }}$ for the overlap region.

(2) Evaluation of $V_{\text {ox }}$ for channel and overlap regions. Here, we consider different operating regions of the device.

(i) $V_{g}<0$.

This region may be further subdivided into two intervals:

(a) $V_{\mathrm{FB}}<V_{g}<0$, and
(b) $-V_{d d}<V_{g}<V_{\mathrm{FB}}$

In region (a), the substrate is in depletion and the electric field across the oxide is not favorable for the electrons to tunnel from poly. But at the two overlap regions, the electric field is directed such that electrons tunnel from the accumulated poly-gate into the overlap region. This is the region where tunneling current is dominated by the edge-component only and it is the principal component of offstate power dissipation in modern day $I C$ chips. In region (b), the channel component becomes comparable and may even dominate over the edge component.

(ii) $V_{g}>0$.

Here the SDE region goes into accumulation and the substrate region goes into inversion beyond $V_{\text {th }}$, hence both the channel component and the edge component are present. The oxide voltage drops for the different gate bias range are given as follows:

$$
V_{\mathrm{ox}}=\left\{\begin{array}{l}
\left(V_{g}-\phi_{s}-V_{\mathrm{FB}}\right) \text { for } V_{g}<0 \\
\left(V_{g e}-\phi_{s}-V_{\mathrm{FB}}\right) \text { for } V_{g}>0
\end{array},\right.
$$

where $\phi_{s}$ is the voltage drop in the semiconductor. Depend- 
ing on whether the semiconductor is in accumulation or depletion/inversion, we get two different expressions for $\phi_{s}$ lumped into the following equation:

$$
\phi_{s}=\left\{\begin{array}{l}
{\left[\frac{\gamma}{2}\left(-1+k_{1}\right)\right]^{2} \text { for depletion/inversion }} \\
{\left[\frac{\left(V_{g}-V_{\mathrm{FB}}\right)-\beta}{1-\frac{q}{k T} \beta}\right] \text { for accumulation, }}
\end{array}\right.
$$

where

$$
\begin{aligned}
& k_{1}=\sqrt{1+\frac{4\left(V_{g}-V_{g e}-V_{\mathrm{FB}}\right.}{\gamma^{2}}}, \\
& \beta=\frac{T_{\mathrm{ox}} \sqrt{2 \varepsilon_{\mathrm{Si}} k T N_{\mathrm{sub}}}}{\varepsilon_{\mathrm{ox}}},
\end{aligned}
$$

and

$$
\gamma=\frac{T_{\mathrm{ox}} \sqrt{2 \varepsilon_{\mathrm{Si}} q N_{\mathrm{sub}}}}{\varepsilon_{\mathrm{ox}}} .
$$

The sign of $\phi_{s}$ as inducted in Eq. (7) above depends on the direction of band bending in the substrate/overlap.

It is to be noted that depletion approximation has been assumed when the semiconductor is either in depletion or inversion. For accumulation, the exponential nature of charge buildup with respect to the surface potential in $\mathrm{Si}$ is approximated by a first order term. The error incurred is low since band bending in accumulation is generally very small for low voltages and high doping concentration. $N_{\text {sub }}$ is replaced by $N_{\mathrm{ov}}$ whenever the SDE/overlap region is under consideration.

All the calculations have been done assuming $V_{d s}=0$. For nonzero $V_{d s}$, Eq. (1) in our analysis needs to be modi-

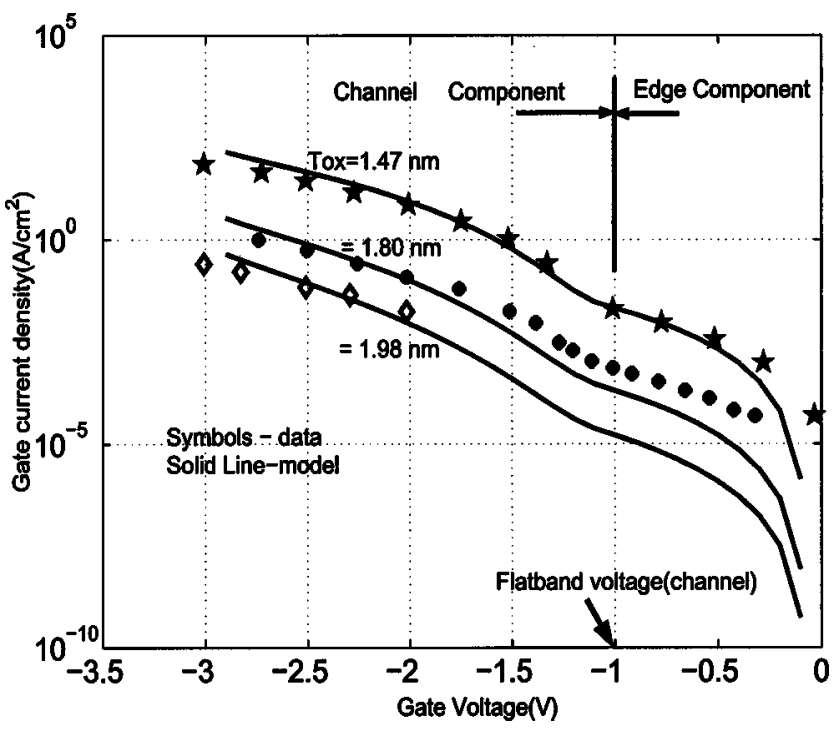

FIG. 2. Proposed model vs data published in Ref. 1 for negative $V_{g}$, the edge and channel component regions are clearly demarcated. A constant overlap length of $20 \mathrm{~nm}$ is used.

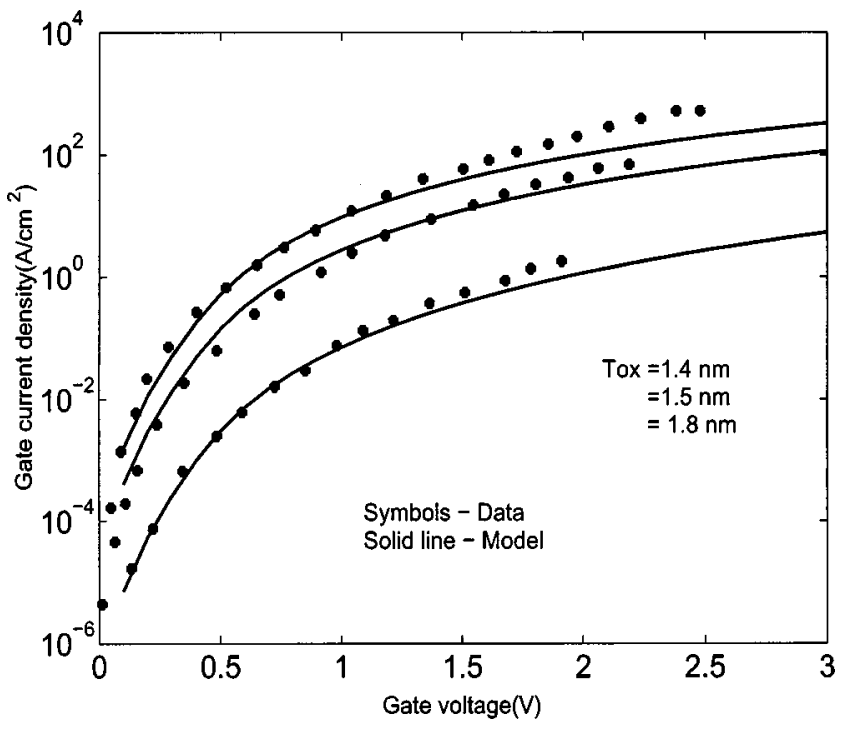

FIG. 3. Proposed model vs data published in Ref. 1 for positive $V_{g}$.

fied to include the channel component and one edge component which depends on $V_{g s}$ and the other edge component which depends on $V_{g d}$.

\section{RESULTS AND DISCUSSION}

Figure 2 shows the comparison between the tunneling current magnitudes calculated by the proposed scheme and data published in Ref. 1 for a $4.65 \mu \mathrm{m}$ nMOSFET with 20 $\mathrm{nm}$ overlap for negative gate voltage. The kink observable around $V_{\mathrm{FB}}=-1.0 \mathrm{~V}$ marks the transition from the EDT dominated tunneling regime to the channel-component dominated regime. Figure 3 shows the comparison between the proposed model and the data for positive gate voltage. It can be seen that the model prediction matches very well with experimental data.

Figure 4 illustrates the prediction of the proposed mod-

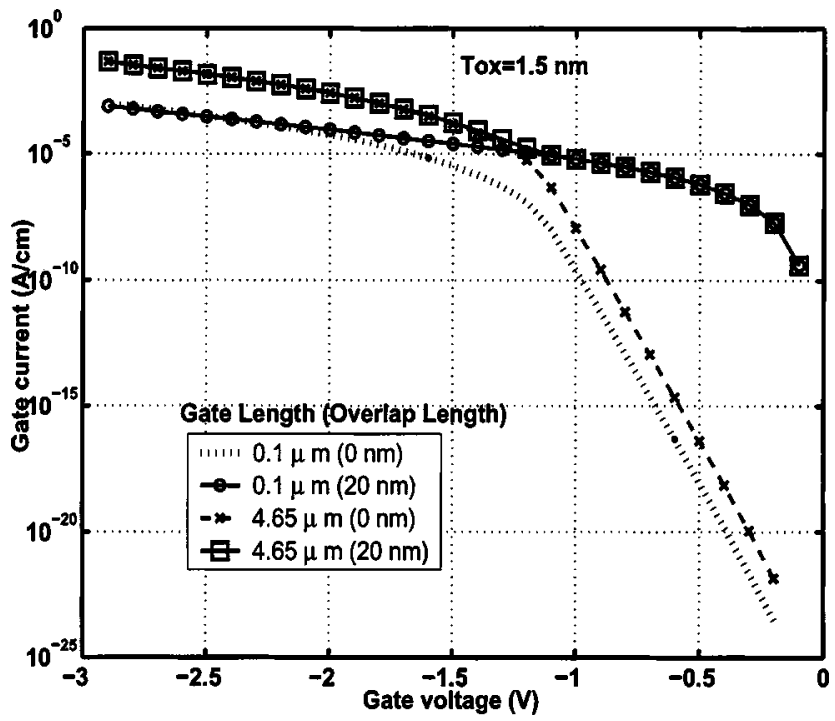

FIG. 4. Simulated tunneling current trends for negative $V_{g}$ for two different channel lengths for a fixed oxide thickness when overlap length is varied from 0 to $20 \mathrm{~nm}$. 


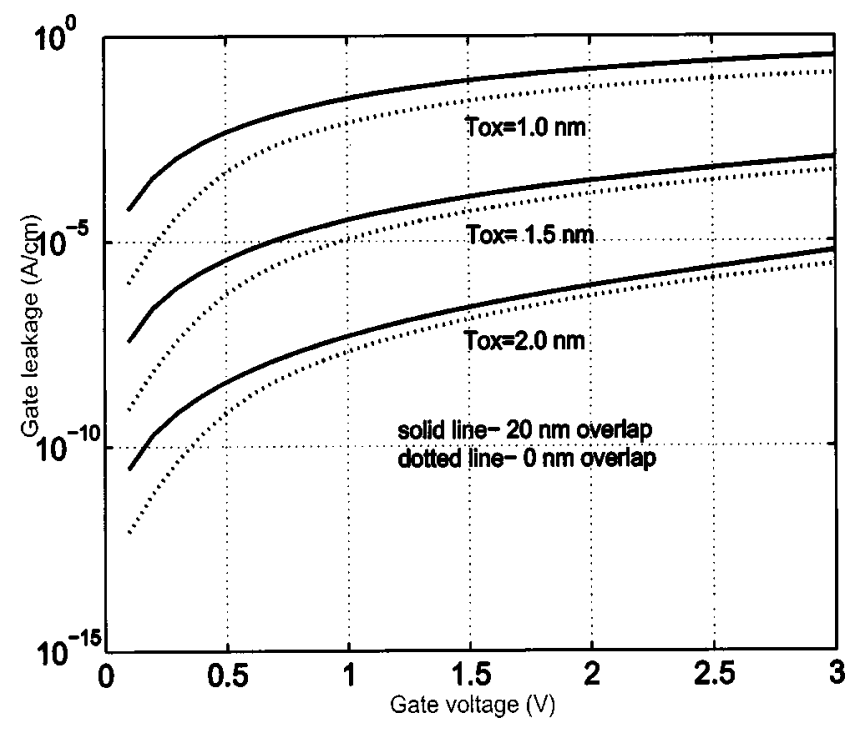

FIG. 5. Simulated tunneling current trends with oxide thickness scaling for a $100 \mathrm{~nm}$ transistor for two different overlap lengths.

eling technique for different poly-Si gate lengths. As the channel length is shrunk from 4.65 to $0.1 \mu \mathrm{m}$, for a $20 \mathrm{~nm}$ overlap, the kink seen in the $I_{g}-V_{g}$ characteristics disappears. This is due to the fact that now the edge component is comparable to the channel component at this dimension. Further in the edge component dominated region, the currents are comparable for both gate lengths since the channel contribution can be neglected.

In Fig. 5, the scalability of overlap length in the context of gate oxide leakage for positive $V_{g}$ has been emphasized. For lower oxide thickness, the difference between tunneling current per unit length for 0 and $20 \mathrm{~nm}$ overlap length for a $100 \mathrm{~nm}$ transistor becomes more pronounced.

The impact of overlap length on the magnitude of tunneling current is better illustrated in Fig. 6. The tunneling current at gate voltage of $1.2 \mathrm{~V}$ (intended supply voltage) is plotted as a function of overlap length. The leakage currents

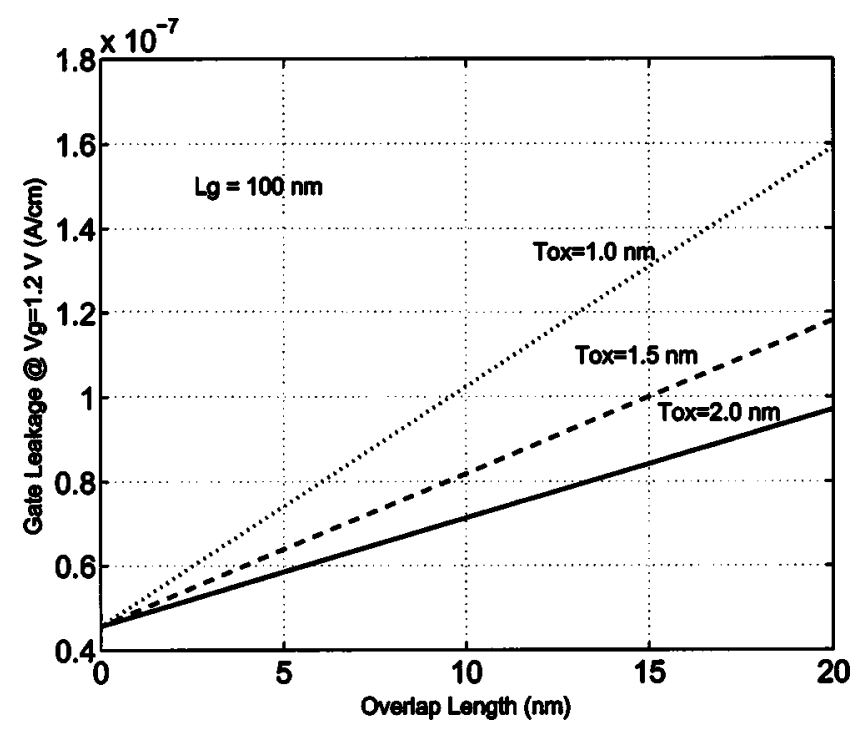

FIG. 6. Tunneling current trends for different oxide thicknesses for varying overlap lengths (values normalized to $T_{\mathrm{ox}}=2.0 \mathrm{~nm}$ ).

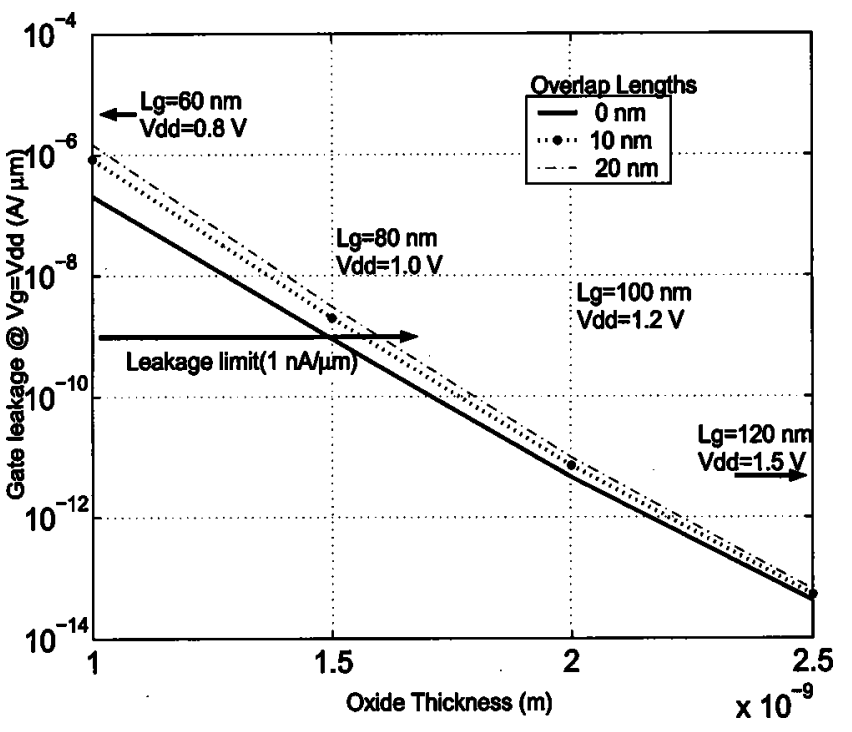

FIG. 7. Scalability of overlap length with respect to gate oxide leakage for variable gate lengths and supply voltages.

for three different oxide thicknesses are matched at $0 \mathrm{~nm}$ by normalization. The increase in leakage current for any given oxide thickness is evident. The effect of overalp length on scaling with the leakage constraint of $1 \mathrm{nA} / \mu \mathrm{m}$ has been demonstrated in Fig. 7. The different technology nodes are illustrated with the appropriate combination of gate length, oxide thickness, and supply voltage. The overlap length influences the scaling limit on oxide thickness to achieve a certain leakage current specification.

\section{CONCLUSIONS}

We have proposed a simple, fully analytical framework, which combines the channel and the edge component of direct tunneling. With gate leakage impacting circuit performance, it is necessary to come up with schemes to model the direct tunneling component which will be simple enough, yet reasonably accurate.

The relative ease of handling this model compared to more computationally complex gate leakage models makes it a possible framework for integrating with circuit simulators. The simple analytical scheme has been shown to predict tunneling leakge fairly accurately for both positive and neagtive gate biases.

Lastly, we have also shown that ignoring the edge component even for positive $V_{g}$ leads to significant inaccuracies. The scalability of gate leakage with overlap length needs to be taken into account particularly in those applications where leakage requirements are very restrictive. It is desirable to minimize the overlap length for minimizing static power consumption through gate direct tunneling.

${ }^{1}$ N. Yang, W. K. Hensen, and J. J. Wortman, IEEE Trans. Electron Devices 47, 1636 (2000)

${ }^{2}$ K. N. Yang, H. T. Huang, M. J. Chen, Y. M. Lin, M. C. Yu, S. M. Jang, D. C. H. Yu, and M. S. Liang, IEEE Trans. Electron Devices 48, 1159 (2001).

${ }^{3}$ N. Yang, W. K. Hensen, J. R. Hauser, and J. J Wortman, IEEE Trans. Electron Devices 46, 1464 (1999).

${ }^{4}$ W-C. Lee and C. Hu, IEEE Trans. Electron Devices 48, 1366 (2001).

${ }^{5}$ S. Thompson, P. Packan, T. Ghani, M. Stettler, M. Alavi, I. Post, S. Tyagi, 
S. Ahmed, S. Yang and M. Bohr, Symposium on VLSI Technology Digest of Technical Papers (IEEE, New York, 1998), pp. 132 and 133.

${ }^{6}$ K. F. Schuegraf, C. C. King, and C. Hu, Digest Symposium VLSI (IEEE, New York, 1992), pp. 18 and 19.
${ }^{7}$ Khairurrijal, W. Mizubayashi, S. Miyazaki, and M. Hirose, J. Appl. Phys. 87, 3000 (2000).

${ }^{8}$ L. F. Register, E. Rosenbaum, and K. Yang, Appl. Phys. Lett. 74, 457 (1999). 